\title{
Novel Targeted Therapies for Patients with Ovarian Cancer Samir A Farghaly*
}

Joan and Sanford I. Weill Medical College/ The Graduate School of Medical Sciences, The New York Presbyterian Hospital-Weill Cornell Medical Center, Cornell University, New York, NY-USA

The number of ovarian cancer new cases diagnosed worldwide each year is 225,000 , with 140,000 related deaths. Early diagnosis of this disease is challenging because of the non-specific symptoms presented during the early stages of the disease. Fifty percent of cases present with advanced metastatic cancer, and the 5-year survival rates are $10-30 \%$. The disease recurrence is high, and the survival rate is only $30 \%$ patients who are sensitive to platinum-based chemotherapy. The increased characterization of the molecular mechanisms involved in the development and progression of ovarian cancer, has resulted in improved therapeutic strategies with molecular-targeted agents. Clinical trials data are accruing for antiangiogenic therapy, including vascular endothelial growth factor (VEGF)-specific inhibitors and multiple angiogenic signaling target inhibitors, and poly-ADP-ribose polymerase (PARP) inhibitors. Other types of pathway inhibitors, including those that target phosphatidylinositol-3-kinase (PI3K), mammalian target of rapamycin (mTOR), protein kinase B (AKT), Src, folate receptor alpha, and insulin-like growth factor-1 receptor (IGF$1 \mathrm{R})$ pathways are in different phases of development for ovarian cancer. Agents that inhibit the epidermal growth factor receptor (EGFR) of ovarian tumors show promise. Moreover an inhibitor of Wee-1 in p53-deficient tumors is being evaluated in recurrent ovarian cancer. Ultimately, Percision therapy using novel biomarkers with improved early detection techniques may markedly enhance the prognosis of ovarian cancer patients.

In this special issue of the Journal of Cancer Sciences and Therapy, various approaches to molecular targeted therapy for ovarian cancer are presented. Uddin et al. discussed the mitochondrial biogenesis (MtBIO) as a target for ovarian cancer therapy through overcoming resistance. Elit and Hirte reviewed various compounds including antibodies that target growth factors receptors or ligands for receptors, small molecules that inhibit tyrosine kinase activities or regulate DNA repair mechanism for ovarian cancer molecular therapy. Mubuchi et al. presented the scientific rationale and evidence for potential clinical benefits provided by mammalian target of rapamycin (mTOR) inhibitors in patients with epithelial ovarian cancer. Lee et al. discussed RP215 and GHR106 monoclonal antibodies as immunotherapeutic treatment ovarian cancer. Ricci et al. presented the role of cancer stem cells and epithelial to mesenchymal transition (EMT) in determining recurrence in ovarian cancer. Nakamura et al. investigated, in ovarian cancer cell lines, the efficacy of local adenovirus-mediated VASH1 gene transfer for anti-angiogenic ovarian cancer treatment. Toss and Cortesi detailed the molecular mechanisms of poly (ADP-ribose) polymerase inhibitors (PARP) in BRCA related ovarian cancer.
*Corresponding author: Samir A Farghaly, Joan and Sanford I. Weill Medical College/ The Graduate School of Medical Sciences, The New York Presbyterian Hospital-Weill Cornell Medical Center, Cornell University, New York, NY-USA, Tel: (206) 203-4905; E-mail: samirfarghaly@yahoo.com

Received November 06, 2014; Accepted November 10, 2014; Published November 14, 2014

Citation: Farghaly SA (2014) Novel Targeted Therapies for Patients with Ovarian Cancer. J Cancer Sci Ther 6: e133. doi:10.4172/1948-5956.1000e133

Copyright: (c) 2014 Farghaly SA. This is an open-access article distributed under the terms of the Creative Commons Attribution License, which permits unrestricted use, distribution, and reproduction in any medium, provided the original author and source are credited. 